\title{
Statistical simulations of the dust foreground to cosmic microwave background polarization (Corrigendum)
}

\author{
F. Vansyngel ${ }^{1}$, F. Boulanger ${ }^{1}$, T. Ghosh ${ }^{1,2}$, B. Wandelt ${ }^{3,4}$, J. Aumont ${ }^{1}$, A. Bracco $^{1,5}$, F. Levrier $^{6}$, \\ P. G. Martin ${ }^{7}$, and L. Montier ${ }^{8}$ \\ ${ }^{1}$ Institut d'Astrophysique Spatiale, CNRS, Université Paris-Sud, Université Paris-Saclay, Bât. 121, 91405 Orsay Cedex, France \\ ${ }^{2}$ California Institute of Technology, Pasadena, CA 91125, USA \\ ${ }^{3}$ Sorbonne Universités, UPMC Université Paris 6 et CNRS, UMR 7095, Institut d'Astrophysique de Paris, 98 bis bd Arago, \\ 75014 Paris, France \\ ${ }^{4}$ Sorbonne Universités, Institut Lagrange de Paris (ILP), 98 bis Boulevard Arago, 75014 Paris, France \\ ${ }^{5}$ Laboratoire AIM, IRFU/Service d'Astrophysique, CEA/DSM, CNRS, Université Paris Diderot, Bât. 709, CEA-Saclay, \\ 91191 Gif-sur-Yvette Cedex, France \\ ${ }^{6}$ LERMA, Observatoire de Paris, PSL Research University, CNRS, Sorbonne Universités, UPMC Université Paris 06, \\ Ecole normale supérieure, 75005, Paris, France \\ ${ }^{7}$ CITA, University of Toronto, 60 St. George St., Toronto, ON M5S 3H8, Canada \\ ${ }^{8}$ CNRS, IRAP, 9 Av. Colonel Roche, BP 44346, 31028 Toulouse Cedex 4, France
}

A\&A, 603, A62 (2017), https://doi.org/10.1051/0004-6361/201629992

Key words. polarization - ISM: general - comic background radiation - submillimeter: ISM - errata, addenda

The caption of Fig. 8 had a confusing description of the material shown in the plots. The correct caption together with the figure is given below.

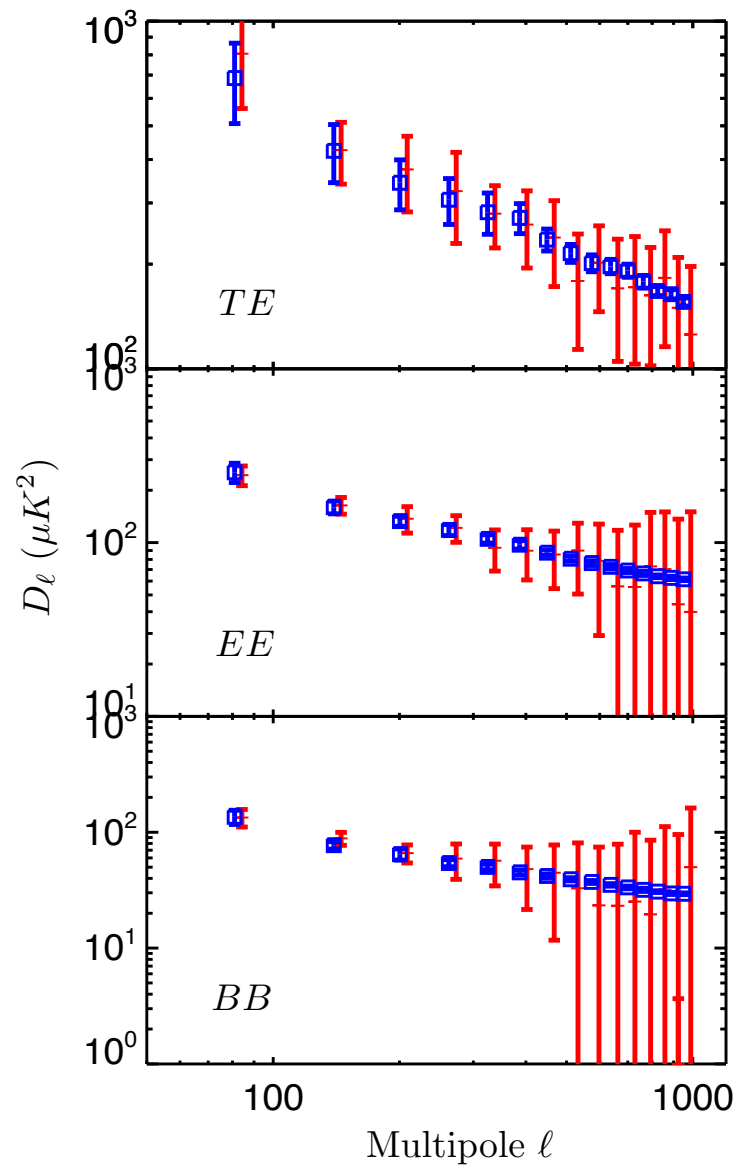

Fig. 8. High-resolution power spectra on the LR63 region; simulation vs. data. From top to bottom: TE, EE, and $B B$ power spectra of the Planck $353 \mathrm{GHz}$, CMB-corrected maps (red crosses), and one highresolution $\left(N_{\text {side }}=2048, F W H M=10^{\prime}\right)$ realization of the model (blue squares). 